\title{
Model theory and modules
}

Prest, Mike

2003

MIMS EPrint: 2006.112

Manchester Institute for Mathematical Sciences

School of Mathematics

The University of Manchester

\footnotetext{
Reports available from: http://eprints.maths.manchester.ac.uk/

And by contacting: The MIMS Secretary

School of Mathematics

The University of Manchester

Manchester, M13 9PL, UK
}

ISSN 1749-9097 


\title{
Model Theory and Modules
}

\author{
Mike Prest, \\ Department of Mathematics \\ University of Manchester \\ Manchester M13 9PL \\ UK \\ mprest@ma.man.ac.uk
}

May 22, 2006

The model-theoretic investigation of modules has led to ideas, techniques and results which are of algebraic interest, irrespective of their model-theoretic significance. It is these aspects that I will discuss in this article, although I will make some comments on the model theory of modules per se.

Our default is that the term "module" will mean (unital) right module over a ring (associative with 1 ) $R$. The category of such modules is denoted Mod- $R$, the full subcategory of finitely presented modules will be denoted mod- $R$, the notation $R$-Mod denotes the category of left $R$-modules. By $\mathbf{A b}$ we mean the category of abelian groups.

In Part 1 we introduce the general concepts and in Part 2 we discuss these in more specific contexts.

References within the text, as well as those in the bibliography, are neither complete nor comprehensive but are intended to lead the reader to a variety of sources.

\section{1}

Purity Purity (pure embeddings, pure-injective modules) undoubtedly plays the central role so we will start with that. The notion of a pure embedding between modules was introduced by Cohn [30]. We say that the module $A$ is a pure submodule of the module $B$ if every finite system $\sum_{i} x_{i} r_{i j}=a_{j}$ $(j=1, \ldots, m)$ of $R$-linear equations with constants from $A$ (so $r_{i j} \in R$ and $a_{j} \in A$ ) and with a solution in $B$ has a solution in $A$ (a solution being elements $b_{1}, \ldots, b_{n}$ such that $\sum_{i} b_{i} r_{i j}=a_{j}$ for all $j$ ). We extend this in the obvious way to define the notion of a pure embedding between modules and we also say 
that an exact sequence $0 \longrightarrow A \stackrel{f}{\longrightarrow} B \longrightarrow C \longrightarrow 0$ is pure-exact if $f$ is a pure embedding.

Functor categories Let $D(R)=(R$-mod, $\mathbf{A b})$ denote the category of additive functors (from now on we use the term "functor" to mean additive functor) from the category of finitely presented left modules to the category of abelian groups. This is a Grothendieck abelian category. It has a generating set consisting of finitely presented objects: indeed, the representable functors $(L,-)$ for $L \in R$-mod are the finitely generated projective objects and, together, are generating. This category is locally coherent - any finitely generated subfunctor of a finitely presented functor is itself finitely presented - and of global dimension less than or equal to 2. A functor is finitely presented iff it is the cokernel of a map between two representable functors. The full subcategory $C(R)=(R \text {-mod, } \mathbf{A b})^{\mathrm{fp}}$ of finitely presented functors is an abelian category and the inclusion of $(R \text {-mod, } \mathbf{A b})^{\mathrm{fp}}$ into $(R$-mod, $\mathbf{A b})$ preserves exact sequences.

Notice that the category $(R$-mod, $\mathbf{A} \mathbf{b})$ is just the category of "modules" over the "ring with many objects" $R$-mod (better, over a small version of this). Concepts for modules over a ring generally make good sense in this context and largely can be understood in this way (that is, as having the same content that they have for modules).

There is a full embedding of Mod- $R$ into $(R$-mod, $\mathbf{A b})$ which is given on objects by sending $M \in \operatorname{Mod}-R$ to the functor $M \otimes_{R}-: R$-mod $\longrightarrow \mathbf{A b}$ and which is given in the natural way on morphisms. The image of this embedding consists (up to isomorphism) of the absolutely pure objects of $(R$-mod, $\mathbf{A b})$. A module $M$ is said to be absolutely pure (=fp-injective) if every embedding $M \longrightarrow$ $M^{\prime}$ in Mod- $R$ is pure (equivalently if whenever $A \stackrel{f}{\rightarrow} B$ is an embedding of modules such that $B / f A$ is finitely presented then each morphism $A \stackrel{g}{\rightarrow} M$ can be factored, through $f$, as $g=h f$ for some $B \stackrel{h}{\rightarrow} M$ ) and the same definition may be made for functors. (Indeed, everything that we do here for modules can be done in the setting of any locally finitely presented Grothendieck category and, to some extent, beyond (e.g. see [14], [82]).)

An object $E$ of an abelian category is injective if every inclusion of the form $E \longrightarrow F$ in the category is split. Every object of a Grothendieck category has an injective hull (a "smallest" injective object containing it).

Theorem 1.1 The following are equivalent for the exact sequence $0 \longrightarrow A \stackrel{f}{\longrightarrow}$ $B \longrightarrow C \longrightarrow 0$ of right $R$-modules:

(i) the sequence is pure-exact;

(ii) for every (finitely presented) left module $L$ the sequence $0 \longrightarrow A \otimes_{R} L \longrightarrow$ $B \otimes_{R} L \longrightarrow C \otimes_{R} L \longrightarrow 0$ of abelian groups is exact;

(iii) for every (finitely presented) left module $L$ the morphism $A \otimes_{R} L \stackrel{f \otimes_{R} 1_{L}}{\longrightarrow}$ $B \otimes_{R} L$ of abelian groups is monic; 
(iv) for every finitely presented module $M$ the sequence $0 \longrightarrow(M, A) \longrightarrow$ $(M, B) \longrightarrow(M, C) \longrightarrow 0$ is exact.

(v) the natural transformation $f \otimes_{R}-: A \otimes_{R}-\longrightarrow B \otimes_{R}-$ is a monomorphism in $(R$-mod, $\mathbf{A b})$.

Proposition 1.2 Every split embedding is pure. The composition of two pure embeddings is pure. If $A \stackrel{f}{\rightarrow} B$ is a pure embedding and if the cokernel $B / f A$ is finitely presented then $f$ is a split embedding.

So purity is significant in the presence of "large" (non-finitely presented) modules. Here is another indication of this.

Proposition 1.3 Any direct limit of pure embeddings is pure.

In particular, any direct limit of split embeddings, though not necessarily split, will be pure.

Pure-injectives A module $N$ is pure-injective (also called algebraically compact) if whenever $N \stackrel{f}{\rightarrow} M$ is a pure embedding then $f$ is split. If we think of pure embeddings as embeddings which would split if the smaller module had "enough" elements then we can see pure-injectivity as a kind of completeness property. 
pp-definable subgroups Let $G=\left(r_{i j}\right)_{i j}$ be a matrix with entries in $R$. Let $M$ be any module. Then $\operatorname{ann}_{M^{n}} G=\left\{\bar{c} \in M^{n}: \sum c_{i} r_{i j}=0\right.$ for each $\left.j\right\}$ is an $\operatorname{End}(M)$-submodule of $M^{n}$ (where $\operatorname{End}(M)$ acts diagonally on $M^{n}$ ) and the projection of $\operatorname{ann}_{M^{n}} G$ to the first, say, $k$ coordinates is a subgroup of, even an $\operatorname{End}(M)$-submodule of, $M^{k}$. Such subgroups are termed variously finitely matrisable subgroups ([181]), subgroups of finite definition ([54]) or, as we shall say, pp-definable subgroups.

Notice that the subgroup of $M^{k}$ that we just defined consists of all $k$-tuples $\bar{a}=\left(a_{1}, \ldots, a_{k}\right)$ from $M$ such that there exists a tuple, $\bar{b}=\left(b_{1}, \ldots, b_{n-k}\right)$, of elements from $M$ such that $(\bar{a} \bar{b}) G=0$ (where $(\bar{a} \bar{b})$ denotes the row vector $\left.\left(a_{1}, \ldots, a_{k}, b_{1}, \ldots, b_{n-k}\right)\right)$. That is, it is the subgroup $\left\{\bar{a} \in M^{k}: M \models\right.$ $\left.\exists y_{1}, \ldots, y_{n-k} \bigwedge_{j} \sum_{i=1}^{k} a_{i} r_{i j}+\sum_{l=1}^{n-k} y_{l} r_{k+l, j}=0\right\}$ of $M^{k}$. Here one should read the symbol $\models$ as "satisfies the condition that" and $\bigwedge$ denotes repeated conjunction ("and"). If we denote by $\phi\left(x_{1}, \ldots, x_{k}\right)$ the condition (on $x_{1}, \ldots, x_{n}$ ) $\exists y_{1}, \ldots, y_{n-k} \bigwedge_{j} \sum_{i=1}^{k} x_{i} r_{i j}+\sum_{l=1}^{n-k} y_{l} r_{k+l, j}=0$ then we may regard this as a formula of the formal language which is used for the model theory of modules. A formula of this particular shape is referred to as a positive primitive (or pp) formula. More loosely, any formula which is equivalent in all $R$-modules to one of this form is said to be a pp formula.

The above subgroup of $M^{k}$ consists of all $\bar{a}$ which satisfy the formula $\phi$ (we write $M \models \phi(\bar{a})$ for that) and we denote this "solution set" of $\phi$ in $M$ by $\phi(M)$. That explains the terminology "pp-definable subgroup".

Observe that, having specified the matrix $G$ and the integer $k$ (and hence the formula $\phi$ above), we obtain, by the above construction, a functor, $F_{\phi}$, from Mod- $R$ to $\mathbf{A b}$ (indeed, a subfunctor of the representable functor $\left.(R,-)^{k}\right)$ : namely that which takes a module $M$ to the group $\phi(M)$ (the action on morphisms is restriction/corestriction since if $A \stackrel{f}{\rightarrow} B$ is any morphism of modules then $f \phi(A) \subseteq \phi(B))$. Using the fact that such a functor commutes with direct limits, together with the fact that every module is a direct limit of finitely presented modules, one sees that this functor is determined by its restriction, which we also denote $F_{\phi}$, to mod- $R$.

If $\phi, \psi$ are pp formulas we write $\psi \leq \phi$ if $\psi$ implies $\phi$, that is, if $\psi(M) \leq \phi(M)$ for every (finitely presented) module $M$, that is, if $F_{\psi}$ is a subfunctor, $F_{\psi} \leq F_{\phi}$, of $F_{\phi}$.

Theorem 1.4 (e.g. [102, Chapter 12]) Every functor of the form $F_{\phi}$ is finitely presented in $(\bmod -R, \mathbf{A b})$. Every finitely presented functor in $(\bmod -R, \mathbf{A b})$ is isomorphic to one the form $F_{\phi} / F_{\psi}$ for some pp formulas with $\psi \leq \phi$.

Theorem 1.5 (e.g. [69, 7.1]) For a module $N$ the following are equivalent:

(i) $N$ is pure-injective;

(ii) any system $\sum_{i \in I} x_{i} r_{i j}=a_{j}(j \in J)$ of $R$-linear equations in possibly infinitely many variables $x_{i}$ (but with, for each $j$, almost all $r_{i j}$ zero) which is finitely solvable in $N$ (i.e. for every finite $J^{\prime} \subseteq J$ the system $\sum_{i \in I} x_{i} r_{i j}=a_{j}$ 
$\left(j \in J^{\prime}\right)$ has a solution in $\left.N\right)$ has a solution in $N$;

(iii) if $C_{k}(k \in K)$ is any set of cosets of pp-definable subgroups of $N$ with the finite intersection property (i.e. for every finite subset $K^{\prime} \subseteq K$ the intersection $\bigcap_{k \in K^{\prime}} C_{k}$ is non-empty) then it has non-empty intersection: $\bigcap_{k \in K} C_{k} \neq \emptyset$; (iv) $N$ is injective over pure embeddings (if $A \stackrel{f}{\rightarrow} B$ is a pure embedding and if $A \stackrel{g}{\rightarrow} N$ is any morphism then there is a factorisation of $g$ through $f$ );

(v) the functor $N \otimes_{R}-$ is an injective object of the functor category (R-mod, $\left.\mathbf{A b}\right)$; (vi) For every index set I the summation map $N^{(I)} \longrightarrow N$, given by $\left(a_{i}\right)_{i \in I} \mapsto$ $\sum_{i} a_{i}$, factors through the canonical embedding of the direct sum $N^{(I)}$ into the direct product $N^{I}$.

Examples 1.6 Any injective module is pure-injective. Any module which is artinian over its endomorphism ring is pure-injective (this includes finite modules and modules which are finite-dimensional over a field contained in the centre of $R$ ). If $M_{R}$ is a module then the left $R$-module $\operatorname{Hom}_{\mathbb{Z}}(M, \mathbb{Q} / \mathbb{Z})$ is pure-injective.

Linear and algebraic compactness The module $M$ is said to be linearly compact if every set of cosets of submodules of $M$ which has the finite intersection property has non-empty intersection. So if $M$ is an $R$-module then $M$ is a pure-injective $R$-module if it is linearly compact as a module over its endomorphism ring (or any subring thereof).

In general pure-injective modules are not closed under extensions (e.g. [143, p. 436]) but one has the useful result [177]: if $0 \longrightarrow A \longrightarrow B \longrightarrow C \longrightarrow 0$ is an exact sequence with $A$ and $C$ linearly compact then $B$ is linearly compact.

Pure-injective hulls Every module $M$ has a pure-injective hull, which we denote variously as $\bar{M}$ or $P I(M): M$ purely embeds, $M \stackrel{j}{\rightarrow} \bar{M}$, in $\bar{M} ; \bar{M}$ is a pure-injective module; if $M \stackrel{f}{\rightarrow} N$ is a pure embedding of $M$ into a pureinjective module $N$ then there is a morphism (necessarily a pure embedding) $\bar{M} \stackrel{g}{\rightarrow} N$ with $g j=f$. This module, $\bar{M}$, is unique up to isomorphism over $M$. It is most efficiently produced (at least, given the corresponding theorem for injective objects of Grothendieck categories) using the following result (take the injective hull of the functor $M \otimes_{R}-$ ), as is the structure theorem that follows.

Theorem 1.7 ([54]) Every injective object of the functor category (R-mod, Ab) has the form $N \otimes_{R}$ - for some pure-injective module $N$.

Theorem 1.8 Let $N$ be a pure-injective module. Then $N \simeq P I\left(\bigoplus_{\lambda} N_{\lambda}\right) \oplus N_{c}$ where each $N_{\lambda}$ is an indecomposable pure-injective and where $N_{c}$ is a continuous (=superdecomposable) pure-injective (that is, one without any non-zero indecomposable summands). The modules $N_{\lambda}$, together with their multiplicities, as well as the module $N_{c}$, are determined up to isomorphism by $N$.

The next result has been extensively used in the model theory of modules. 
Theorem 1.9 ([44], also see [47]) Let $N$ be a pure-injective module and let $A$ be a submodule of $N$. Then there is a direct summand of $N$, denoted $H(A)$, which is determined up to isomorphism over $A$ and which is minimal in the sense that if $A \leq N^{\prime}$ and $N^{\prime}$ is a direct summand of $N$ then there is an A-isomorphism of $H(A)$ with a direct summand of $N^{\prime}$.

In fact, $H(A)$ may be identified as that module such that $H(A) \otimes_{R}-$ is the injective hull of the image of $A \otimes_{R}-$ in $N \otimes_{R}-$.

$\Sigma$-pure-injectives and modules of finite endolength Any direct product of pure-injective modules is pure-injective. A module $M$ is said to be $\Sigma$-pureinjective if $M^{\left(\aleph_{0}\right)}$ (and hence every direct sum of copies of $M$ ) is pure-injective.

Theorem 1.10 A module $M$ is $\Sigma$-pure-injective iff $M$ has the descending chain condition on pp-definable subgroups. In particular, any module which is artinian over its endomorphism ring is $\Sigma$-pure-injective.

Theorem 1.11 If $M$ is $\Sigma$-pure-injective then so is every pure submodule of $M$ (hence such a module is a direct summand) as is every module in the closure of $\{M\}$ under arbitrary direct sums, direct products, direct limits and direct summands.

Every $\Sigma$-pure-injective module is a direct sum of indecomposable pure-injective modules.

A module $M$ is of finite endolength if it is of finite length when considered as a module over its endomorphism ring. Such a module must be $\Sigma$-pure-injective.

Theorem 1.12 ([47]) The module $M$ is of finite endolength iff for any index set $I$, the power $M^{I}$ is a direct sum of copies of direct summands of $M$.

A module which is of finite endolength and is not finitely presented is said to be generic. At least in the context of finite-dimensional algebras over algebraically closed fields, such modules correspond to one-parameter families of finite-dimensional modules, see [32] and, for more general contexts, [34].

The next result indicates the model-theoretic relevance of pure-injectivity and its second part points to the special role played by the indecomposable pure-injectives.

Theorem 1.13 ([43], [151]) Every module is elementarily equivalent to a pureinjective module (in fact, is an elementary substructure of its pure-injective hull).

([178]) Every module is elementarily equivalent to a direct sum of indecomposable pure-injectives (for elementary equivalence see below). 
pp-types There are various ways in which one may try to explain the key role played by pure-injective modules in the model theory of modules. One is to say that they are the "positively saturated" modules - those which realise every pp-type. Saturation is a notion, a kind of completeness property, from model theory and the proofs of many results in model theory involve moving to a suitable saturated elementary extension. The analogous procedure here is moving to the pure-injective hull (or some other pure-injective pure extension) of a module. The notion of a pp-type has played a central role in the modeltheoretic approach to modules yet its algebraic translation is not a familiar algebraic object. I now say something about this.

Let $a \in M$. The pp-type of $a$ in $M$ is just the set of pp formulas satisfied by $a$ in $M: \operatorname{pp}^{M}(a)=\{\phi(x): M \models \phi(a)\}$. If we replace each pp formula by the corresponding subfunctor, $F_{\phi}$, of the forgetful functor, $(R,-) \in(\bmod -R, \mathbf{A b})^{\mathrm{fp}}$, then $\mathrm{pp}^{M}(a)$ becomes a filter in the lattice of finitely generated subfunctors of $(R,-)$. If $M$ is finitely presented then this filter will be principal, that is there will be some pp formula $\phi_{0} \in \operatorname{pp}^{M}(a)$ which implies all the rest (said in terms of functors, $\phi_{0}$ is such that the intersection of this filter is $F_{\phi_{0}}$ and $F_{\phi_{0}}$ belongs to the filter). I remark that the formula $\phi_{0}$ can be computed explicitly from a presentation of $M$ by generators and relations and the representation of $a$ as an $R$-linear combination of these generators.

We can make rather more algebraic sense of this by moving to the dual functor category $(R \text {-mod, } \mathbf{A b})^{\mathrm{fp}}$ (see the subsection on duality of functors). In that context the element $a \in M$, that is the morphism $R \stackrel{a}{\rightarrow} M$, becomes the morphism $(R R,-) \simeq\left(R \otimes_{R}-\right) \stackrel{a \otimes-}{\longrightarrow}(M \otimes-)$ in $(R$-mod, Ab) and the "duals", $d F_{\phi}$, in the sense discussed below, of the functors $F_{\phi}$ with $\phi \in \operatorname{pp}^{M}(a)$ turn out to be exactly the finitely generated subfunctors of the kernel of $a \otimes-$. That is, the pp-type of $a$ in $M$ is "really" the kernel of the morphism $a \otimes-:(R \otimes-) \longrightarrow$ $(M \otimes-)$. In [102] the pp-type of an element was treated heuristically as a generalised annihilator of the element. The above change of perspective makes this precise.

Ziegler spectrum The (right) Ziegler spectrum, $\mathrm{Zg}_{R}$, of $R$ is the topological space which has, for its points, the (isomorphism types of) indecomposable pureinjectives and which has, for a basis of open sets, those given by:

pairs of pp-formulas - let $\phi \geq \psi$ be pp formulas (in any number of free variables) and set

$(\phi / \psi)=\left\{N \in \mathrm{Zg}_{R}: \phi(N) / \psi(N) \neq 0\right\}$

alternatively, by finitely presented functors in $(R$-mod, $\mathbf{A b})$ - let $F \in(R \text {-mod, } \mathbf{A b})^{\mathrm{fp}}$ and set $(F)=\left\{N \in \mathrm{Zg}_{R}:(F, N \otimes-) \neq 0\right\}$

alternatively by morphisms in mod- $R$ - let $A \stackrel{f}{\rightarrow} B$ be a morphism in mod- $R$ and set $(f)=\left\{N \in \mathrm{Zg}_{R}:(f, N):(B, N) \longrightarrow(A, N)\right.$ is not onto $\}$.

These are, indeed, equivalent: any set of one of the above forms is of the other 
forms (and the transformation from one to the other form is quite explicit and can be found variously in [36], [77], [115]). Since every functor in (mod- $R, \mathbf{A b})^{\mathrm{fp}}$ is isomorphic to $F_{\phi} / F_{\psi}$ for some pp formulas $\phi \geq \psi$ a fourth way of giving the open sets is as

$\left\{N \in \mathrm{Zg}_{R}: \vec{G} N \neq 0\right\}$ as $G$ ranges over (mod- $\left.R, \mathbf{A b}\right)^{\mathrm{fp}}$ and where $\vec{G}$ is the unique extension of such a functor to a functor on all of Mod- $R$ which commutes with direct limits.

Theorem 1.14 ([178, 4.9]) The space $\mathrm{Zg}_{R}$ is compact, that is, every open cover of $\mathrm{Zg}_{R}$ has a finite subcover. The compact open sets are precisely the basic open sets (variously) described above.

Ziegler showed that the closed subsets of this space correspond exactly to those elementary classes of modules which are closed under direct sum and direct summand (Crawley-Boevey, [36], has termed such classes definable, which is a useful term although it has potential to be confused with the more general "axiomatisable").

Support of a module The support of a module $M$ is $\operatorname{supp}(M)=\{N \in$ $\mathrm{Zg}_{R}: N$ is a direct summand of $M^{\prime}$ for some $M^{\prime} \equiv M$ \}. (Modules $M, M^{\prime}$ are elementarily equivalent (see [116]), $M \equiv M^{\prime}$, if they have exactly the same first order properties - a first order property being one which can be expressed by a sentence, or set of sentences, of the formal language for $R$-modules. It is equivalent to require that they have isomorphic ultrapowers.) This is a closed subset of $\mathrm{Zg}_{R}$ and every closed subset is the support of some module. One has $\operatorname{supp}(M)=\operatorname{supp}\left(M_{1}\right)$ iff $M^{\left(\aleph_{0}\right)} \equiv M_{1}^{\left(\aleph_{0}\right)}$ (if, for instance, $R$ is an algebra over an infinite field then one has $\operatorname{supp}(M)=\operatorname{supp}\left(M_{1}\right)$ iff $\left.M \equiv M_{1}\right)$. One can avoid the use of elementary equivalence in this definition by working with torsion theories in the functor category, as follows.

To each module $M$ is associated a hereditary torsion theory (for torsion theory see [162]) on the functor category ( $R$-mod, $\mathbf{A b}$ ): namely that cogenerated by $E(M \otimes-)=\bar{M} \otimes-$. So the corresponding torsion class is $\mathcal{T}=\{F \in$ $(R$-mod, $\mathbf{A b}):(F, M \otimes-)=0\}$. Denote the associated torsion theory by $\tau_{M}$. This is a torsion theory of finite type since the torsion class is determined by (is the smallest hereditary torsion class containing) the finitely presented torsion functors. It is the case that $\tau_{M}$ is cogenerated by a set of indecomposable injective objects. The set of points $N \in \mathrm{Zg}_{R}$ such that $N \otimes-$ is $\tau_{M}$-torsionfree is the support of $M$ as defined above.

If $X$ is any closed subset of $\mathrm{Zg}_{R}$ and if $\tau=\tau_{X}$ is the corresponding torsion theory of finite type on $(R$-mod, $\mathbf{A b})$ (that is, the torsion theory cogenerated by $\{N \otimes-: N \in X\}$ ) then the modules $M$ with $\operatorname{supp}(M) \subseteq X$ are exactly those such that $M \otimes-$ is $\tau$-torsionfree. One also has, for any module $M$, that $\operatorname{supp}(\bar{M} / M) \subseteq \operatorname{supp}(M)=\operatorname{supp}(\bar{M})$ and so, if $\operatorname{supp}(M) \subseteq X$, then $(\bar{M} / M) \otimes-$ is $\tau$-torsionfree and hence $M \otimes-$ is $\tau$-closed (in the sense 
of torsion theory). Indeed, see [55], [62], [74], the functors $M \otimes-$ for $M$ with $\operatorname{supp}(M) \subseteq X$ give exactly the absolutely pure objects of the category $(R \text {-mod, } \mathbf{A b})_{\tau}$ where by $(R \text {-mod, } \mathbf{A} \mathbf{b})_{\tau}$ we mean the category which is obtained by localising $(R$-mod, $\mathbf{A b})$ at $\tau$.

Isolated points A point, $N$, of $\mathrm{Zg}_{R}$ is isolated (that is $\{N\}$ is open) iff there is a finitely presented functor $F$ from $R$-mod to $\mathbf{A b}$ such that $(F, N \otimes-) \neq 0$ and such that $N$ is the only such indecomposable pure-injective. For instance, if $F$ is a finitely presented simple object of $(R$-mod, $\mathbf{A b})$ then $(F)$ contains just one point, namely the point $N \in \mathrm{Zg}_{R}$ such that $N \otimes-$ is the injective hull of $F$. We say that $N \in \mathrm{Zg}_{R}$ is isolated by a minimal pair if $\{N\}=(\phi / \psi)$ for some pair, $\phi>\psi$ of pp formulas such that no pp formula lies strictly between $\phi$ and $\psi$. A morphism $f: A \longrightarrow B$ is said to be minimal left almost split if it is not a split monomorphism but every morphism $g: A \longrightarrow C$ which is not a split monomorphism factors through $f$ and if every endomorphism $h$ of $B$ with $h f=f$ is an automorphism of $B$ (see [5]).

Theorem 1.15 (a) Suppose that $N \in \mathrm{Zg}_{R}$. Then $N$ is isolated by a minimal pair iff $N \otimes-$ is the injective hull of a finitely presented simple functor in $(R$-mod, $\mathbf{A b})$.

(b) If $N \in \mathrm{Zg}_{R}$ is the pure-injective hull of a finitely presented module $M$ then $N$ is isolated by a minimal pair iff there is a minimal left almost split map in $\bmod -R$ with source $M$ (see, e.g., [62, 7.7]).

(c) If $R$ is countable and $N \in \mathrm{Zg}_{R}$ is isolated then $N$ is isolated by a minimal pair ([178]).

It is not known whether or not the equivalence in part (c) holds for general rings. It does hold whenever the functor category $(R$-mod, Ab) has KrullGabriel dimension (see below).

Related to the above one has the following.

Theorem 1.16 ([34, 2.3], also see [182]) An indecomposable pure-injective module is the injective hull of a simple functor iff it is the source of a left almost split map in Mod-R.

Theorem 1.17 ([102, §11.3], [59]) Let $M \in \bmod -R$. Then the pure-injective hull, $\bar{M}$, of $M$ is indecomposable iff $\operatorname{End}(M)$ is local.

Theorem 1.18 ([62, Prop. 5.4]) Let $R$ be a ring such that every finitely presented module is a direct sum of modules with local endomorphism ring. Then the points of $\mathrm{Zg}_{R}$ of the form $\bar{M}$ where $M$ is finitely presented and indecomposable are dense in $\mathrm{Zg}_{R}$. If mod-R has almost split sequences then all such points are isolated by minimal pairs (e.g. [120, Prop. 3.7]).

In particular, if $R$ is an artin algebra then every indecomposable finitely generated $R$-module is an isolated point of $\mathrm{Zg}_{R}$ and, together, these points are dense in $\mathrm{Zg}_{R}$ [102, Chapter 13]. 
Closed points As for closed points, we have the following.

Theorem 1.19 If $M \in \operatorname{Mod}-R$ is of finite endolength then $\operatorname{supp}(M)$ is a finite set of closed points of $\mathrm{Zg}_{R}$. In particular, if $N$ is indecomposable and of finite endolength then $N$ is a closed point of $\mathrm{Zg}_{R}$. For countable rings $R$ we have the converse: if $N$ is a closed point of $\mathrm{Zg}_{R}$ then $N$ is of finite endolength.

It is not known whether or not the last part holds for arbitrary rings. It does hold if ( $R$-mod, Ab) has Krull-Gabriel dimension (by the analysis of [178], see, e.g., [102, Section 10.4]).

Finiteness conditions A central aim is to understand the Ziegler spectrum: to prove general results and to obtain descriptions of $\mathrm{Zg}_{R}$ (points and topology) for particular rings $R$. There are "finiteness conditions" and related dimensions and ranks which aid the analysis of $\mathrm{Zg}_{R}$. Here we discuss the Krull-Gabriel and uniserial dimensions of the functor category ( $R$-mod, $\mathbf{A b})$ and the CantorBendixson rank of $\mathrm{Zg}_{R}$ (also see [110], [115]). Both dimensions on the functor category are obtained by successive localisation.

Krull-Gabriel dimension Let $\mathcal{C}$ be a locally coherent Grothendieck category, such as $(R$-mod, $\mathbf{A b})$. If $\tau$ is a torsion theory of finite type on $\mathcal{C}$ then the quotient category $\mathcal{C}_{\tau}$ is again locally coherent (with finitely presented objects exactly the objects isomorphic to localisations of finitely presented objects of C).

Denote by $\mathcal{S}_{0}$ the subcategory of $\mathcal{C}^{\text {fp }}$ (the full subcategory of finitely presented objects of $\mathcal{C}$ ), consisting of all finitely presented objects of finite length. This is a Serre subcategory of $\mathcal{C}^{\mathrm{fp}}$ (if $0 \longrightarrow A \longrightarrow B \longrightarrow C \longrightarrow 0$ is exact then $B \in \mathcal{S}_{0}$ iff $\left.A, C \in \mathcal{S}_{0}\right)$ and hence its closure under direct limits is the torsion class for a torsion theory, $\tau=\tau(\mathcal{C})$, of finite type on $\mathcal{C}$. The corresponding quotient category $\mathcal{C}^{(1)}=\mathcal{C}_{\tau}$ is obtained from $\mathcal{C}$ by "making zero" all finitely presented simple objects. Since this localised category is again locally coherent Grothendieck ([62], [74], [104]) we can repeat the process.

Having defined $\mathcal{C}^{(\alpha)}$ we define $\mathcal{C}^{(\alpha+1)}$ to be $\mathcal{C}_{\tau(\mathcal{C}(\alpha))}^{(\alpha)}$. The process can be continued transfinitely as follows. Let $\tau_{\alpha}$, with corresponding torsion class denoted $\mathcal{T}_{\alpha}$, be the torsion theory on $\mathcal{C}$ such that $\mathcal{C}^{(\alpha)}=\mathcal{C}_{\tau_{\alpha}}$. If $\lambda$ is a limit ordinal define $\tau_{\lambda}$ to be the torsion theory which has torsion class $\bigcup\left\{\mathcal{T}_{\alpha}: \alpha<\lambda\right\}$ and define $\mathcal{C}^{(\lambda)}$ to be the localisation of $\mathcal{C}$ at $\tau_{\lambda}$.

The least $\alpha$ such that $\mathcal{C}^{(\alpha)}=0$ (that is, such that $\mathcal{T}_{\alpha}=\mathcal{C}$ ) is the KrullGabriel dimension, $\operatorname{KGdim}(\mathcal{C})$, [48] of $\mathcal{C}$ : if there is no such $\alpha$ (that is, if some non-zero localisation of $\mathcal{C}$ has no finitely presented simple object) then we set $\operatorname{KGdim}(\mathcal{C})=\infty$ (and say that the Krull-Gabriel dimension of $\mathcal{C}$ is "undefined"). The Krull-Gabriel dimension of an object $C \in \mathcal{C}$ is the least $\alpha$ such that the image of $C$ in $\mathcal{C}^{(\alpha)}$ is zero (that is, $C \in \mathcal{T}_{\alpha}$ ), if this exists, and is $\infty$ otherwise. We set $\operatorname{KG}(R)=\operatorname{KGdim}(R$-mod, $\mathbf{A b})$ and refer to this also as the Krull-Gabriel dimension of $R$. 
Theorem 1.20 $\mathrm{KG}(R)<\infty$ iff the lattice of finitely generated subfunctors of the functor $\left({ }_{R} R,-\right) \in(R$-mod, $\mathbf{A b})$ has Krull dimension in the sense of Gabriel-Rentschler.

m-dimension Recall that Krull dimension (in the sense of [137]) is defined on posets by inductively collapsing intervals which have the descending chain condition. One can define a variant of this dimension by inductively collapsing intervals of finite length. This dimension, called "m-dimension" in [102], does, therefore, grow more slowly than Krull dimension but the one dimension is defined iff the other is (iff the poset contains no densely ordered sub-poset) Actually, the discussion in [102] is in terms of the lattice of pp formulas but this is isomorphic to the above lattice of finitely generated functors via the map $\phi \mapsto F_{\phi}$. So we have $\mathrm{m}$-dim $(M)=\operatorname{KGdim}(M)$ for every module $M$.

Recall that if $X$ is a closed subset of $\mathrm{Zg}_{R}$ then the modules $M$ with $\operatorname{supp}(M) \subseteq$ $X$ correspond to the absolutely pure objects of the localised category $(R \text {-mod, } \mathbf{A} \mathbf{b})_{\tau}$ where $\tau=\tau_{X}$ is the torsion theory of finite type on $(R-\bmod , \mathbf{A b})$, which we also denote $D(R)$, corresponding to $X$. In particular, the points, $N$, of $X$ correspond to the indecomposable injectives, $(N \otimes-)_{\tau} \simeq(N \otimes-)$, of $(R \text {-mod, } \mathbf{A b})_{\tau}$.

Theorem 1.21 Suppose that $X$ is a closed subset of $\mathrm{Zg}_{R}$, let $\tau$ be the corresponding torsion theory on $(R-\bmod , \mathbf{A b})$ and let $(R-\bmod , \mathbf{A b})_{\tau}$ be the localisation of $(R$-mod, $\mathbf{A b})$ at this torsion theory.

If $\operatorname{KGdim}\left((R-\bmod , \mathbf{A b})_{\tau}\right)=\alpha<\infty$ then every pure-injective module with support contained in $X$ is the pure-injective hull of a direct sum of indecomposables ([47]). Furthermore, the Cantor-Bendixson rank of $X$ equals $\alpha$ ([178]). In particular, the isolated points of $X$ are dense in $X$.

In particular if $\mathrm{KG}(R)<\infty$ then there are no continuous pure-injective $R$-modules.

Cantor-Bendixson rank The Cantor-Bendixson rank of a topological space $T$ is defined as follows. Let $T^{\prime}$ be the set of non-isolated points of $T$. Inductively set $T^{(0)}=T, T^{(\alpha+1)}=\left(T^{(\alpha)}\right)^{\prime}, T^{(\lambda)}=\bigcap\left\{T^{(\alpha)}: \alpha<\lambda\right\}$ for limit ordinals $\lambda$. The Cantor-Bendixson rank, $\mathrm{CB}(p)$, of a point $p \in T$ is the least $\alpha$ such that $p \notin T^{(\alpha)}$, and is $\infty$ if there is no such $\alpha$. The Cantor-Bendixson rank, $\mathrm{CB}(T)$, of $T$ is the least $\alpha$ such that $T^{(\alpha)}=\emptyset$ if such exists, and is $\infty$ otherwise. If $T$ is a compact space then $\mathrm{CB}(T)=\max \{\mathrm{CB}(p): p \in T\}$ and there are only finitely many points of maximum CB-rank if this is less than $\infty$.

Isolation condition We say that $R$ satisfies the isolation condition if for every closed subset $X$ of $Z_{R}$ every point $N \in X$ which is isolated in $X$ is isolated by an $X$-minimal pair, meaning that there are $\psi \leq \phi$ with $\{N\}=X \cap(\phi / \psi)$ and such that the localised functor $\left(F_{\phi} / F_{\psi}\right)_{\tau=\tau_{X}}$ is a simple object of $(R \text {-mod, } \mathbf{A b})_{\tau}$ (an equivalent condition on this pair is that for any/every $M$ with $\operatorname{supp}(M)=X$ 
we have $\phi(M)>\psi(M)$ and there is no pp-definable subgroup of $M$ strictly between $\phi(M)$ and $\psi(M)$ ). (In [102] this condition was given the ad hoc name "condition $(\wedge)$ ".) The condition is right/left symmetric (it holds for $R$ iff it holds for $\left.R^{\mathrm{op}}\right)$. It is not known whether or not every ring satisfies the isolation condition (in the case of von Neumann regular rings this specialises to an existing open question about such rings, see subsection $6 \mathrm{~b}$ below).

Theorem 1.22 ([178]) Every countable ring satisfies the isolation condition. If $\operatorname{KG}(R)<\infty$ or, more generally, if there are no continuous pure-injective $R$-modules then $R$ satisfies the isolation condition.

Theorem 1.23 If $R$ satisfies the isolation condition then $\mathrm{CB}\left(\mathrm{Zg}_{R}\right)=\mathrm{KG}(R)$. In particular this is true if $R$ is countable.

Uniserial dimension The other dimension that we consider is obtained by the same general process that we used to define Krull-Gabriel dimension. If $\mathcal{C}$ is a locally finitely presented Grothendieck category let $\mathcal{S}_{u}$ denote the Serre subcategory of $\mathcal{C}^{\mathrm{fp}}$ which is generated by the finitely presented uniserial objects (an object is uniserial if the lattice of its subobjects is a chain). The same process of successive localisation that we used for Krull-Gabriel dimension now yields the notion of uniserial dimension ( $\infty$ or "defined" - that is, an ordinal) of a locally coherent Grothendieck category. We write $\operatorname{UD}(R)$ for the uniserial dimension of the functor category $(R$-mod, Ab). Clearly $\mathrm{UD}(R) \leq \operatorname{KGdim}(R)$.

Theorem 1.24 [178] If the uniserial dimension of $(R-\bmod , \mathbf{A b})$ is defined then there are no continuous pure-injective $R$-modules. If $R$ is countable then the converse is true.

It is not known whether or not the converse is true for all rings.

Gabriel-Zariski spectrum Next we consider a new topology on the set of isomorphism classes of indecomposable pure-injective $R$-modules. Consider the collection, $\left\{U^{c}: U\right.$ a compact open subset of $\left.\mathrm{Zg}_{R}\right\}$, of complements of compact Ziegler-open sets. We use the notation $[\phi / \psi],[F]$ for the complements of $(\phi / \psi)$, $(F)$ respectively. This collection of sets is closed under finite intersection and so forms a basis for a new topology: the dual-Ziegler, or Zariski, topology on $\mathrm{Zg}_{R}$. We will call the resulting topological space the Gabriel-Zariski spectrum, $\operatorname{Zar}_{R}$, of $R$ and also the Zariski spectrum of mod- $R$.

We explain the terminology. Let $R$ be a commutative noetherian ring. Recall that the Zariski spectrum of $R$ is the set, $\operatorname{spec}(R)$, of prime ideals of $R$ equipped with the topology which has, for a basis of open sets, those of the form $\{P \in$ $\operatorname{spec}(R): r \notin P\}$ as $r$ varies over $R$. Following Gabriel and Matlis [45], [90] we replace each prime $P$ by the injective hull, $E(R / P)$, of the factor module $R / P$. This is an indecomposable injective $R$-module (denote the set of isomorphism classes of these by $\operatorname{Inj}_{R}$ ) and every indecomposable injective $R$-module has this 
form. To any finitely presented module $M$ associate $\left\{E \in \operatorname{Inj}_{R}: \operatorname{Hom}(M, E)=\right.$ $0\}$. These sets, as $M$ varies, form a basis of a topology on $\operatorname{Inj}_{R}$. Indeed, the resulting space is homeomorphic to $\operatorname{spec}(R)$ via the identification of $P$ with $E(R / P)$. Thus $\operatorname{spec}(R)$ may be defined purely in terms of the module category.

This definition makes sense in any locally coherent category, in particular, in the category $D(R)=(R$-mod, $\mathbf{A b})$. Since the indecomposable injective objects of $D(R)$ correspond bijectively with the indecomposable pure-injective right $R$ modules this topology on $D(R)$ induces one on the set $\mathrm{Zg}_{R}$. This new topology on the set $\mathrm{Zg}_{R}$ is exactly the one we defined above, the basic open sets having the form $\{N:(F, N \otimes-)=0\}$ as $F$ ranges over the subcategory $C(R)=$ $(R \text {-mod, } \mathrm{Ab})^{\mathrm{fp}}$, hence the name.

Despite the name, however, the space $\operatorname{Zar}_{R}$ is only "algebraic-geometric" in parts. For example it is seldom compact and it may have infinitely many clopen points. In some examples (see, e.g., [27]) it seems to be a partial amalgamation of "geometric" and "combinatorial" pieces.

Nonetheless, there is a natural sheaf of rings over it which directly generalises the structure sheaf of a commutative noetherian ring. We need the notion of the ring of definable scalars in order to define this.

Rings of definable scalars Let $X$ be a closed subset of $\mathrm{Zg}_{R}$. Let $M$ be a module with $\operatorname{supp}(M)=X$. Then the set of pp-definable functions from $M$ to $M$ forms what is called the ring of definable scalars, $R_{M}$, of $M$. This ring, rather $R$-algebra since there is a canonical morphism $R \longrightarrow R_{M}$, depends only on $X$, so we denote it also by $R_{X}$ Every module with support contained in $X$ is naturally an $R_{X}$-module. This ring may be defined in other ways, without direct reference to model theory, as follows.

First, choose $M$ with $\operatorname{supp}(M)=X$ to be pure-injective and also such that $M \otimes-$ is an injective cogenerator for the torsion theory of finite type, $\tau$, on $D(R)$ which corresponds to $X$. Then $R_{X}$ is the biendomorphism ring, $\operatorname{Biend}(M)$, of $M^{I}$ (that is, $\operatorname{End}\left(\operatorname{End}(M) M^{I}\right)$ ) where the power $I$ is chosen large enough so that $M^{I}$ is cyclic over its endomorphism ring.

Alternatively, consider the localisation, $\left({ }_{R} R,-\right)_{\tau}$, of the forgetful functor at the torsion theory $\tau=\tau_{\operatorname{supp}(M)}$. Then the endomorphism ring, in the localised category, $D(R)_{\tau}$, of $\left({ }_{R} R,-\right)_{\tau}$ is isomorphic to $R_{X}$.

Theorem 1.25 (a) [26] Let $M$ be a $\Sigma$-pure-injective module which is finitely generated over its endomorphism ring (e.g. let $M$ be a module of finite endolength). Then $R_{M} \simeq \operatorname{Biend}(M)$.

(b) [108] Suppose that $R \stackrel{f}{\rightarrow} S$ is an epimorphism in the category of rings. Then the forgetful functor from Mod-S to Mod- $R$ induces an embedding of $\mathrm{Zg}_{S}$ as a closed subset of $\mathrm{Zg}_{R}$. The ring of definable scalars of this closed subset is exactly $S$, regarded as an $R$-algebra via $f$.

(c) [104] Let $E$ be an injective $R$-module which cogenerates a torsion theory, $\tau$, of finite type on Mod- $R$. Then the ring of definable scalars of $E$ is precisely the 
corresponding localisation $R \longrightarrow R_{\tau}$.

A finer topology Burke [21] introduced another topology on the underlying set of $\mathrm{Zg}_{R}$ which he (re-)named, in [22], the full support topology (in his thesis he called it the "types-over-formulas" topology because the basic open sets are of the form $(p / \psi)$ where $p$ is a pp-type and $\psi$ a pp formula). The closed sets for this topology are exactly the sets of the form $\{N: N \otimes-$ is $\tau$-torsionfree $\}$ where now $\tau$ ranges over all hereditary torsion theories (not just those of finite type) on $(R$-mod, $\mathbf{A b})$. Associated to any closed set of this topology is the ring of type-definable scalars, which is isomorphic to the endomorphism ring of the localised forgetful functor. Corresponding to this topology one also obtains a sheaf of rings, with stalks being rings of infinitely definable scalars, analogous to that defined below.

The sheaf of locally definable scalars For every basic closed subset $X$ of $\mathrm{Zg}_{R}$ we have the associated $R$-algebra, $R \longrightarrow R_{X}$, of definable scalars. These sets form a basis of open sets for $\operatorname{Zar}_{R}$ and so this assignment defines a presheaf on this basis of $\operatorname{Zar}_{R}$, hence extends to a sheaf on $\operatorname{Zar}_{R}$, called the sheaf of locally definable scalars, $\operatorname{LDef}_{R}$, of $R$. One can check that the stalk of this sheaf at an indecomposable injective $N$ is just $R_{N}$. Such a ring need not be local but its centre will be and so the centre of $\operatorname{LDef}_{R}$ is a sheaf of commutative local rings.

As remarked already this is the usual definition of structure sheaf for a commutative noetherian ring extended to a more general context. One can also check that, for such a ring $R$, the restriction of $\operatorname{LDef}_{R}$ to the (Ziegler-closed) subset, $\operatorname{Inj}_{R}$, of $\mathrm{Zg}_{R}$ is just the usual structure sheaf of $R$.

In fact, the sheaf of locally definable scalars is just a part of a richer structure. Consider the presheaf which assigns to a closed subset $X$ of $\mathrm{Zg}_{R}$ the corresponding localisation, $(R \text {-mod, } \mathbf{A b})_{\tau}^{\mathrm{fp}}=\left((R \text {-mod, } \mathbf{A b})_{\tau}\right)^{\mathrm{fp}}$ where $\tau=\tau_{X}$, of the subcategory of finitely presented objects of the functor category. This sheaf of skeletally small abelian categories is denoted $\mathbf{L D e f}_{R}$ and has a natural interpretation in model-theoretic terms, as the sheaf of categories of imaginaries associated with the category of $R$-modules [113].

Duality of functors Next we turn to duality between right and left modules. The basic duality, which is valid for all $\operatorname{rings} R$, is between the categories, $C(R)=(R-\bmod , \mathbf{A b})^{\mathrm{fp}}$ and $C\left(R^{\mathrm{op}}\right)=(\bmod -R, \mathbf{A} \mathbf{b})^{\mathrm{fp}}$ of finitely presented functors.

Theorem 1.26 ([4], [55]) For any ring $R$ we have $C\left(R^{\mathrm{op}}\right) \simeq C(R)^{\mathrm{op}}$ via the contravariant functor which is defined on objects by taking $F \in C\left(R^{\mathrm{op}}\right)$ to the functor, $D F$, in $C(R)$ which is given on objects by taking $L \in R$-mod to $(F,-\otimes$ $L)$ (since $L$ is finitely presented one does have $\left.-\otimes L \in(\bmod -R, \mathbf{A b})^{\mathrm{fp}}\right)$. 
The model-theoretic version of this duality ([101]) is that the lattice of pp formulas for right modules is anti-isomorphic to the lattice of $\mathrm{pp}$ formulas for left modules. Equivalently, the lattice of finitely generated subfunctors of the forgetful functor $\left(R_{R},-\right) \in D\left(R^{\mathrm{op}}\right)$ is anti-isomorphic to the lattice of finitely generated subfunctors of the functor $\left({ }_{R} R,-\right) \simeq(R \otimes-,-) \in D(R)$. The correspondence sends an inclusion $F \stackrel{f}{\longrightarrow}\left(R_{R},-\right)$ to $d F=\operatorname{ker}\left(\left({ }_{R} R,-\right) \stackrel{D f}{\longrightarrow} D F\right)$. This was extended by Herzog to give a duality between Ziegler spectra.

Duality of spectra Let $X$ be a closed subset of $\mathrm{Zg}_{R}$. Consider the corresponding Serre subcategory of $C(R)=(R \text {-mod, } \mathbf{A b})^{\mathrm{fp}}, \mathcal{S}_{X}=\{F \in C(R):(F, N \otimes$ $-)=0$ for all $N \in X\}$. The duals, $D F$, of these functors form a Serre subcategory, $D \mathcal{S}_{X}$, of $C\left(R^{\mathrm{op}}\right)$. In fact, we get exactly those functors $G \in C\left(R^{\mathrm{op}}\right)$ such that $\vec{G}(N)=0$ for every $N \in X$. This follows immediately from the formula $(F, M \otimes-) \simeq \vec{F}(M)([103])$ which is valid for any $F \in C(R)$ and $M \in$ Mod- $R$. Denote by $D X$ the closed subset, $\left\{N \in{ }_{R} \mathrm{Zg}:(G,-\otimes N)=0\right.$ for all $\left.G \in D \mathcal{S}_{X}\right\}$, of ${ }_{R} \mathrm{Zg}$ corresponding to $D \mathcal{S}_{X}$.

Recall that the collection of open subsets of any topological space forms a locale (a complete Heyting algebra, that is, a complete lattice in which meet distributes over arbitrary joins).

Theorem 1.27 Let $R$ be any ring. Then the map $X \mapsto D X$ between closed subsets of $\mathrm{Zg}_{R}$ and ${ }_{R} \mathrm{Zg}$ is a bijection which commutes with arbitrary unions and intersections. Thus the locales of open subsets of $\mathrm{Zg}_{R}$ and ${ }_{R} \mathrm{Zg}$ are isomorphic (that is, these spaces are "homeomorphic at the level of topology").

([58, 4.9]) If $R$ is countable then there is actually a homeomorphism (that is, "at the level of points") between $\mathrm{Zg}_{R} / \approx$ and ${ }_{R} \mathrm{Zg} / \approx$ which induces $X \mapsto D X$. $H e r e \approx$ denotes topological equivalence: the equivalence relation which identifies two points if they belong to exactly the same open sets.

If $X$ is a closed subset of $\mathrm{Zg}_{R}$ such that $\mathrm{KGdim}\left((R \text {-mod, } \mathbf{A b})_{\tau}\right)<\infty$ (here $\tau=\tau_{X}$ is the localisation corresponding to $\left.X\right)$ then to every point $N \in X$ there is a uniquely defined point $D N \in D X$ such that this correspondence induces a homeomorphism from $X$ to $D X$.

These results have many corollaries including, as well as equality of various dimensions for right and left modules, those below.

Corollaries 1.28 (a) ([58]) For any ring there is a bijection between definable classes of right and left modules.

(b) If $R$ is a countable ring then there is a continuous pure-injective right module iff there is a continuous pure-injective left module.

(c) $([58,4.10]$, also [34, Section 6]) For any ring $R$ there is a bijection (which preserves endolength) between right and left indecomposable modules of finite endolength. 
Let us make the duality between closed sets more concrete. If $M$ is any $R$-module, $S$ is any subring of $\operatorname{End}(M)$ and $E$ is an injective cogenerator for $S$-Mod then the corresponding dual of $M$ is $M^{*}=\operatorname{Hom}_{S}\left({ }_{S} M,_{S} E\right)$. This has a natural structure as a left $R$-module and, as such, it is pure-injective.

Theorem 1.29 ([180], also see [58]) Let $R$ be a ring, $M \in \operatorname{Mod}-R, S$ a subring of $\operatorname{End}(M), E$ an injective cogenerator for $S$-Mod. Regard $M^{*}=$ $\operatorname{Hom}_{S}\left({ }_{S} M,_{S} E\right)$ as a left $R$-module. Then $\operatorname{supp}\left(M^{*}\right)=D(\operatorname{supp}(M))$.

In some cases a suitable choice of $S$ will give the duality $N \mapsto D N$ as $N \mapsto N^{*}$ for at least certain points of the spectrum. For instance if $R$ is an artin algebra and we take $S$ to be a minimal injective cogenerator for the category of modules over $Z(R) / J(Z(R))$ where $Z(R)$ denotes the centre of $R$ and $J$ denotes the Jacobson radical, then $D N \simeq N^{*}$ for $N$ finitely presented (as well as for some infinite-dimensional modules $N$ ).

\section{2}

Now we give information on the Ziegler spectra of various types of ring.

1. Rings of finite representation type $\mathrm{A}$ ring $R$ is said to be of finite representation type if every $R$-module is a direct sum of indecomposable modules and if there are, up to isomorphism, just finitely many indecomposable $R$-modules. The condition for right modules implies that for left modules. It is equivalent that $\mathrm{KG}(R)=0$ and so, for such rings, $\mathrm{Zg}_{R}$ is a discrete space. It is an open question whether $\mathrm{Zg}_{R}$ being discrete implies that $R$ is of finite representation type (see Section $6 \mathrm{~b}$ below). If $R$ is of finite representation type then every module is $\Sigma$-pure-injective.

2. Pure semisimple rings $\mathrm{A}$ ring $R$ is said to be right pure-semisimple if every right $R$-module is a direct sum of indecomposable modules. In this case every right $R$-module is $\Sigma$-pure-injective (in turn, this condition implies right pure-semisimplicity), $R$ must be right artinian, every indecomposable right $R$ module is finitely generated and there are, up to isomorphism, only finitely many indecomposable right $R$-modules of length $n$ for each natural number $n$ [101], [180]. For such a ring $\mathrm{KG}(R)=\mathrm{CB}\left(\mathrm{Zg}_{R}\right)<\infty$. A ring which is right and left pure-semisimple must be of finite representation type. It is a long-standing open question whether or not every right pure-semisimple ring is of finite representation type. For Artin algbras this was shown to be so by Auslander [3]. Herzog [60] showed that it holds for PI rings. Simson has shown (e.g. see [161]) that the general problem reduces to questions about division algebras.

3. Dedekind domains Let $R$ be a commutative Dedekind domain (in fact, what we say here applies equally well if $R$ is a non-commutative Dedekind 
domain which satisfies a polynomial identity). Then the $R$-modules of finite length are pure-injective and the indecomposable ones are exactly the isolated points of $\mathrm{Zg}_{R}$. The set of isolated points is dense in $\mathrm{Zg}_{R}$ and the other points can be obtained as suitable direct limits or inverse limits of these points. The points of Cantor-Bendixson rank 1 are the "prüfer" modules (the injective hulls of the simple $R$-modules) and their duals, the "adic" modules (the completions of $R$ at non-zero primes). There remains the quotient field of $R$ : this is the unique point of (maximal) rank 2. We have $\mathrm{KG}(R)=\mathrm{CB}\left(\mathrm{Zg}_{R}\right)=2$.

4. Artin algebras The ring $R$ is an artin algebra if its centre is artinian and if $R$ is finitely generated as a module over its centre. Finite rings and algebras which are finite-dimensional over a field are examples. Every module of finite length over such a ring is pure-injective and the existence of Auslander-Reiten sequences for such rings yields that the indecomposable modules of finite length are all isolated points of $\mathrm{Zg}_{R}$. Indeed, these are exactly the isolated points and, together, they are dense in $\mathrm{Zg}_{R}$.

Further description of $\mathrm{Zg}_{R}$ is very much tied up with description of the finitely generated modules and hence with the representation type of $R$. For the remainder of this subsection we assume that $R$ is a finite-dimensional $K$ algebra where $K$ is a field. For the precise definition of domestic, tame and wild we refer to [31] for instance (especially since, although the situation is clearcut for finite-dimensional algebras over algebraically closed fields, it is not clear what the definitions of these terms should be in general, although it is clear that various particular algebras belong to the one category or another).

4a. Domestic algebras A $K$-algebra $R$ is domestic if there are finitely many representation embeddings mod- $R^{\prime} \longrightarrow \bmod -R$ where $R^{\prime}$ is a finite localisation of the polynomial ring $K[X]$ such that, for each integer $d \geq 1$, all but finitely many indecomposable $R$-modules of (K-)dimension $d$ lie (up to isomorphism) in the union of the images of these embeddings. For characterisations of these algebras in terms of generic modules see [32], [34]. Tame hereditary finitedimensional $K$-algebras are examples, as are certain string algebras.

Let $R$ be any tame hereditary finite-dimensional $K$-algebra. So, if $K$ is algebraically closed, then $R$ is Morita equivalent to a finite product of rings, each of which is the path algebra over $K$ of an extended Dynkin quiver (for the general case see [37]). Suppose that $R$ is indecomposable as a ring. Then the Ziegler spectrum of $R$ is, roughly, composed of finitely many generically overlapping copies of Ziegler spectra of Dedekind domains, together with the discretely-indexed families of indecomposable pre-projective and pre-injective modules. One has $\mathrm{KG}(R)=\mathrm{CB}\left(\mathrm{Zg}_{R}\right)=2$. See [111], [144].

For some time all the values of $\mathrm{KG}(R)$ computed for $R$ an artin algebra had been 0 (finite representation type), 2 (tame hereditary and some algebras related by tilting) and $\infty$ (some tame but non-domestic algebras and wild algebras). Then, following work of Schröer [157], the values of $\mathrm{KG}(R)$ and $\mathrm{CB}\left(\mathrm{Zg}_{R}\right)$ were computed ([27], [158]) for a series of domestic string algebras which turn out to 
give all integer values $3 \leq n<\omega$. See the references for details, including the explicit description of the points and the topology, which relies heavily on [143] and [25]. It has been shown by Krause [79] (for finite-dimensional algebras over an algebraically closed field) and Herzog [63] (for artin algebras in general) that there is no artin algebra $R$ with $\mathrm{KG}(R)=1$. Hence every finite value, apart from 1 , occurs as $\operatorname{KG}(R)$ for some artin algebra $R$. The author has conjectured that, for an artin algebra $R$, we have $\mathrm{KG}(R)<\infty$ iff $R$ is of domestic representation type.

4b. Tame algebras A $K$-algebra $R$ is tame if, for each integer $d \geq 1$ there are finitely many representation embeddings mod- $R^{\prime} \longrightarrow \bmod -R$ where $R^{\prime}$ is a finite localisation of the polynomial ring $K[X]$ such that all but finitely many indecomposable $R$-modules of ( $K$-)dimension $d$ lie (up to isomorphism) in the union of the images of these embeddings (but the number of representation embeddings needed might grow as $d$ grows). Examples include string algebras (see [28]) and tame canonical algebras ([141]) and in these examples both $\mathrm{KG}(R)$ and $\mathrm{CB}\left(\mathrm{Zg}_{R}\right)$ turn out to be $\infty$ when the algebra is not domestic. There is still, however, the hope of being able to describe the spectra in these cases (following the idea that if the finite-dimensional modules can be decribed then so can the Ziegler spectrum), indeed, a classification of points in the case of string algebras has been outlined [145] by Ringel. It is also conjectured that if $R$ is a tame algebra then the uniserial dimension of $(R$-mod, $\mathbf{A b})$ is defined and, in particular, that there should be no continuous pure-injective $R$-modules.

4c. Wild algebras A $K$-algebra $R$ is wild if there is a representation embedding from mod- $K\langle X, Y\rangle$, where $K\langle X, Y\rangle$ is the free $K$-algebra on two generators, to mod- $R$. Roughly, this means that there are two-parameter families (and hence $n$-parameter families for each $n$ ) of finite-dimensional $R$-modules and then the classification problem for finite-dimensional $R$-modules is considered to be impossible. For such an algebra we have $\operatorname{KG}(R)=\infty$ and so, at least for countable rings (but conjecturally for all rings), $\mathrm{CB}\left(\mathrm{Zg}_{R}\right)=\infty$. Furthermore the uniserial dimension of $(R-\bmod , \mathbf{A b})$ is $\infty$ and there exist continuous pure-injective $R$-modules.

5. Infinite-dimensional algebras For infinite-dimensional algebras the themes of classification in the tame case and impossibility of complete classification in the wild case continue, even though the terms tame and wild are not generally defined in this context.

5a. Hereditary orders These are not far removed from the tame hereditary finite-dimensional algebras that we discussed above. A ring $R$ is a hereditary order (in a central simple algebra) if $R$ is a hereditary noetherian prime ring which has, for its simple ring of quotients, a matrix ring over a division ring which is finite-dimensional over its centre. Equivalently, $R$ is a hereditary noetherian prime ring which satisfies some polynomial identity. In [33] CrawleyBoevey draws a parallel between and, indeed, links the categories of finite-length modules over a tame hereditary artin algebra and over a hereditary order, with 
the maximal orders corresponding to those artin algebras in which all tubes are homogeneous. Using this, the techniques of [111] which give the description of the Ziegler spectrum of a tame hereditary artin algebra also yield the, almost identical, description for hereditary orders.

5b. Generalised Weyl algebras The first Weyl algebra, $R=K\langle X, Y$ : $X Y-Y X=1\rangle$, over a field, $K$, of characteristic zero is a simple noetherian hereditary domain which is not an order. Klingler and Levy [72] showed that the category of torsion modules over this ring is "wild" and their techniques can be used to show that there is a continuous pure-injective $R$-module. If $M$ is any indecomposable $R$-module of finite length then the pure-injective hull, $\bar{M}$, of $M$ is indecomposable and it follows from a result of Bavula [12] that no such point is isolated (see [120]). In [120] it is shown that the set of points of this form is dense in $\mathrm{Zg}_{R}$ and hence that there are no isolated points in $\mathrm{Zg}_{R}$. These and related results are proved in [120] for a class of rings, certain generalised Weyl algebras in the sense of [11], which includes the first Weyl algebra.

5c. Pullback rings If $R, R^{\prime}$ are two commutative discrete valuation domains and if there is an isomorphism between their residue fields then one may form the pullback in the sense of Levy [84]. An example of a ring so obtained is the algebra $K[X, Y: X Y=0]_{(X, Y)}$ which is the infinite-dimensional version of the GelfandPonomarev algebras $K\left[X, Y: X Y=0=X^{n}=Y^{m}\right]$ which are, for $m, n \geq 2$, $m+n \geq 5$, tame non-domestic string algebras. For such pullback rings Toffalori [168], [169] classified the indecomposable pure-injective "separated" modules and Ebrahimi-Atani [38] classified all the indecomposable pure-injectives, $N$, such that $N / N . J(R)$ is of finite length. Note that the complete description of the Ziegler spectrum for such rings would include that for the tame non-domestic Gelfand-Ponomarev algebras.

5d. Differential polynomial rings Let $K$ be a field and let $d$ be a derivation on $K$ : that is $d: K \longrightarrow K$ is an additive map which satisfies $d(a b)=a . d b+d a . b$. Let $R$ be the corresponding differential polynomial ring: $R$ is the ring of polynomials, with (non-central) coefficients from $K$, in an indeterminate $X$ with relations $a X=X . d a(a \in K)$. By varying $K$ and $d$ we obtain a variety of interesting examples. For instance, suppose that $(K, d)$ is a universal field with derivation. Then $R$ is an example of a V-ring (see [42]) a ring in which every simple module is injective. In this case, there is a unique simple $R$-module, $S$, and the Ziegler spectrum of $R$ consists of just three points: the injective module $S$; the "dual" of this module (the pure-injective hull of $R$ is the pure-injective hull of a direct sum of copies of this dual module); the quotient division ring of $R$. The first two points are isolated (and $S$ is even $\Sigma$-pure-injective), and we have $\mathrm{KG}(R)=\mathrm{CB}\left(\mathrm{Zg}_{R}\right)=1$.

For another class of examples, Puninski [132] investigates the Ziegler spectrum of, and the finite length modules over, the ring of differential operators $\mathcal{D}=K[[X]]\left[\frac{\delta}{\delta X}\right]$ where $K$ is algebraically closed of characteristic zero. The general shape of the spectrum turns out to be similar to that over a commutative Dedekind domain (or over a tame hereditary finite-dimensional algebra). 
In particular the Cantor-Bendixson rank is 2. In [133] he describes the Ziegler spectrum of rings of the form $R=K[X, \delta]$ where $K$ is a field of characteristic 0 , $\delta$ is a derivation on $K$ whose field of constants is algebraically closed and where it is assumed that the category of finite length modules has Auslander-Reiten sequences. Again the description is similar to that seen in the tame hereditary case.

6. Regular rings The ring $R$ is (von Neumann) regular if every finitely generated right (equivalently left) ideal is generated by an idempotent element. The following are equivalent: $R$ is regular; the theory of $R$-modules admits elimination of quantifiers; every pp formula is equivalent to a quantifier-free formula; every module is absolutely pure; every embedding between modules is a pure embedding; every pure-injective module is injective. So for such rings the Ziegler spectrum is the set of isomorphism classes of indecomposable injective modules and the basic open sets are those of the form $\{N$ : $N$ is an indecomposable injective and $N e \neq 0\}$ see [147].

6a. Commutative regular rings The Pierce spectrum of such a ring is the space of maximal ideals equipped with the Stone topology, which has, for a basis of open (and closed) sets, those of the form $\{M: e \notin M\}$ as $e$ ranges over elements of $R$. An injective module is indecomposable iff it is the injective hull of a simple module, and so we have a natural bijection between $\mathrm{Zg}_{R}$ and the Pierce spectrum, which is easily seen to be a homeomorphism (in fact, the Ziegler, Zariski and Pierce topologies coincide). Examples are the boolean rings (that is, boolean algebras): these are the commutative regular rings $R$ such that each factor ring $R / M$, where $M$ is a maximal ideal of $R$, is the field with two elements.

A boolean ring is atomic if every non-zero ideal contains a simple ideal and is superatomic if every factor ring is atomic. The terminology may be extended to general commutative regular rings via their boolean algebras of idempotent elements and then the condition is equivalent to $R$ being semiartinian (that is, every non-zero module has a non-zero socle). Then [47, Theorem 4], for a commutative regular ring $R, R$ is superatomic/semiartinian iff the CantorBendixson rank of $\mathrm{Zg}_{R}$ is defined iff $\mathrm{KG}(R)<\infty$ (and then we have $\mathrm{CB}\left(\mathrm{Zg}_{R}\right)=$ $\mathrm{KG}(R))$. Otherwise, there are continuous (pure-)injective modules, irrespective of the cardinality of the ring. The Pierce sheaf is the sheaf which assigns to the ideal $I$ the factor ring $R / I$. This is naturally identified with the sheaf of locally definable scalars.

6b. (Non-commutative) regular rings Some of the results on commutative regular rings generalise. Trlifaj ([170]) showed that if $R$ is a regular ring then $R$ is semiartinian iff there is no continuous pure-injective $R$-module iff $\mathrm{UD}(R)<\infty$ iff $\mathrm{m}-\operatorname{dim}(R)<\infty$ iff $\operatorname{KG}(R)<\infty$. Also see [171] for $\operatorname{KG}(R)$ and $\mathrm{CB}\left(\mathrm{Zg}_{R}\right)$.

The connection between $\operatorname{KG}(R)$ and $\mathrm{CB}\left(\mathrm{Zg}_{R}\right)$ is, however, open even in this case. Indeed [170] there exists a regular ring $R$ which does not satisfy the 
isolation condition iff there exists a regular ring $R^{\prime}$ which is simple, non-artinian and with $\left|\mathrm{Zg}_{R^{\prime}}\right|=1$. The existence of such a ring $R^{\prime}$ is an open question. One does have [170] that the isolation condition holds for regular rings $R$ which satisfy any of the following conditions: $|R|<2^{\aleph_{0}}$; all primitive factor rings of $R$ are artinian; $R$ is semiartinian.

If $R$ is a regular ring with all primitive factor rings artinian then every indecomposable injective $R$-module is (the injective hull of) a simple module (cf. [53]) and so $\mathrm{Zg}_{R}$ is homeomorphic to the maximal ideal space of $R$. In particular, $\mathrm{Zg}_{R}$ is a $T_{1}$ space. Furthermore if $R$ is regular with all primitive factor rings artinian then the following conditions are equivalent: $\mathrm{Zg}_{R}$ is Hausdorff; $\mathrm{Zg}_{R}$ is a normal space; $\mathrm{Zg}_{R}$ is totally disconnected; $R$ is a biregular ring (for every $x \in R$, the ideal $R x R$ is generated by a central idempotent). The paper [171] contains further results about the relationship between maxspec $(R)$ and $\mathrm{Zg}_{R}$ in the general regular case.

7. Serial rings The model theory of modules over serial rings was investigated by Eklof and Herzog [39] and by Puninski [128]. In both these papers a particularly nice basis of the Ziegler topology was found and general characterisations of indecomposable pure-injectives in terms of the ideals of $R$ were given. Subsequently Puninski [129], [130], in the commutative case, and Reynders [138] have investigated Cantor-Bendixson for Ziegler spectra of serial rings. One has ([130]) that if $R$ is a commutative valuation domain then $\operatorname{Kdim}(R)<\infty$ iff $\mathrm{KG}(R)<\infty$ (and then $\mathrm{KG}(R)=2 \cdot \operatorname{Kdim}(R))$ iff $\mathrm{CB}\left(\mathrm{Zg}_{R}\right)<\infty$ iff there is no continuous pure-injective $R$-module. Furthermore [138] if $R$ is a serial ring with Krull dimension $\alpha<\infty$ then $\operatorname{KG}(R) \leq 2 \alpha$.

Also in [125] it is shown that if $R$ is a commutative valuation domain then there is a superdecomposable pure-injective $R$-module iff the value group of $R$ contains, as a partially ordered subset, a copy of the rationals. See also [131].

In [134] information about the continuous pure-injective modules over a commutative valuation domain is obtained.

8. Pseudo-finite-dimensional representations of $U(\operatorname{sl}(2, K))$ Herzog [65] considers the closure, $C$, in the Ziegler spectrum of $U(\operatorname{sl}(2, K))$ of the finitedimensional representations (regarded as representations of the universal enveloping algebra $U(s l(2, K)))$ of the Lie algebra $s l(2, K)$. By a pseudo-dimensional representation is meant one with support contained in $C$. Herzog shows that the canonical morphism from $U(\operatorname{sl}(2, K))$ to the ring of definable scalars of this set is an epimorphism to a von Neumann regular ring which has the (continuum many) points of $C$ as its simple torsion representations. He also extends some of the theory of weights to these representations.

9. Stable and triangulated categories Benson and Gnacadja [15] show that certain of the idempotent modules of Rickard [139] in the stable module category for a finite group are pure-injective. These results have been extended by Benson 
and Krause [18]. Krause [81] has shown how to define the Ziegler spectrum of any compactly generated triangulated category and in [17] Benson and Krause find the Zariski spectrum (in the classical sense) of the Tate cohomology ring of a finite group as a part of the Zariski spectrum (in the sense used in this paper) of the group ring of that group.

10. Modules over group rings and lattices The model theory of modules over group rings and of lattices over orders has been investigated, in particular by Marcja and Toffalori, especially with a view to showing that the tame/wild dichotomy corresponds to the split between (a ring having) decidable/undecidable theory of modules. See [165], [166] and, for example, [8], [86], [88], [167]. These papers also provide a great variety of examples of interpretations of classes of additive structures in other such classes.

11. Decidability/undecidability The word problem for groups is known to be undecidable: there is no algorithm which, when input with any word $w$ and words $w_{1}, \ldots, w_{n}$ will decide whether or not $w$ represents the identity element in the free group factored by the normal subgroup generated by the words $w_{1}, \ldots, w_{n}$.

Baur [9], [10] and others (for references see [102, Chapter 17]) showed that this unsolvable word problem for groups can be encoded in the theory of modules over various rings. For example the theory of $K\langle X, Y\rangle$-modules, where $K$ is any field, encodes the word problem for groups and hence is undecidable, meaning that there is no algorithm which, input with any sentence from the language of $K\langle X, Y\rangle$-modules, will decide whether or not it is true in all $K\langle X, Y\rangle$-modules.

It has been conjectured by the author that if $R$ is a wild $K$-algebra then the theory of $R$-modules interprets that of $K\langle X, Y\rangle$-modules and hence is undecidable (this is known to be so for strictly wild algebras, see [108]). Indeed all current evidence is in favour of the implication "wild implies undecidable" even outside the context of finite-dimensional algebras, in particular for group rings [165], [166]. The evidence for the implication "tame implies decidable" is rather less compelling but, still, in the light of what is currently known, it seems not unreasonable to conjecture that this is so. Ziegler [178] showed that if enough is known about the topology of the spectrum then one obtains a decision procedure for the theory of modules In all cases where decidability of the theory of modules has been established one has an explicit description of the spectrum. So explicit description of Ziegler spectra also has this application.

\section{References}

[1] J. Adámek and J. Rosický, Locally Presentable and Accessible Categories, London Math. Soc., Lecture Note Ser., Vol. 189, Cambridge University Press, 1994. 
[2] M. Auslander, Coherent functors, pp. 189-231 in Proceedings of the Conference on Categorical Algebra, La Jolla, 1965, Springer-Verlag, 1966.

[3] M. Auslander, Large modules over Artin algebras, pp. 1-17 in Algebra, Topology and Category Theory, Academic Press, New York, 1976.

[4] M. Auslander, Isolated singularities and existence of almost split sequences, (Notes by Louise Unger pp. 194-242 in Representation Theory II, Groups and Orders, Ottawa 1984, Lecture Notes in Mathematics, Vol. 1178, Springer-Verlag, 1986.

[5] M. Auslander, I. Reiten and S. O. Smalø, Representation Theory of Artin Algebras, Cambridge University Press, 1995.

[6] J. T. Baldwin and A. H. Lachlan, On universal Horn classes categorical in some infinite power, Algebra Universalis, 3 (1973), 98-111.

[7] S. Baratella and M. Prest, Pure-injective modules over the dihedral algebras, Comm. Algebra, 25 (1997), 11-32.

[8] S. Baratella and C. Toffalori, The theory of $\mathbb{Z} C(2)^{2}$-lattices is decidable, Arch. Math. Logic, 37 (1998), 91-104.

[9] W. Baur, Decidability and undecidability for theories of abelian groups with predicates for subgroups, Composition Math., 31 (1975), 23-30.

[10] W. Baur, Undecidability of the theory of abelian groups with a subgroup, Proc. Amer. Math. Soc., 55 (1976), 125-128.

[11] V. Bavula, Generalised Weyl algebras and their representations, Algebra and Analiz, 4(1) (1992), 75-97; English transl. in St. Petersburg Math. J., 4(1), (1993), 71-92.

[12] V. Bavula, The extension group of the simple modules over the first Weyl algebra, Bull. London Math. Soc., 32 (2000), 182-190.

[13] V. Bavula and F. van Oystaeyen, The simple modules of certain generalised crossed products, J. Algebra, 194 (1997), 521-566.

[14] A. Beligiannis, Relative homological algebra and purity in triangulated categories, J. Algebra, to appear.

[15] D. Benson and G. Gnacadja, Phantom maps and purity in modular representation theory I, Fund. Math., 161 (1999), 27-91.

[16] D. Benson and G. Gnacadja, Phantom maps and purity in modular representation theory II, Algebras and Representation Theory, to appear. 
[17] D. Benson and H. Krause, Pure injectives and the spectrum of the cohomology ring of a finite group, J. reine angew. Math., to appear.

[18] D. Benson and H. Krause, Generic idempotent modules for a finite group, Algebras and Representation Theory 3 (2000), 337-346.

[19] K. I. Beidar, A. V. Mikhalev and G. Puninski, Logical aspects of ring and module theory, Fundamentalnaya i Prikladnaya Matematika, 1 (1995), 1-62.

[20] Proceedings of the conference "Infinite Length Modules, Bielefeld, 1998", to appear.

[21] K. Burke, Some Model-Theoretic Properties of Functor Categories for Modules, Doctoral Thesis, University of Manchester, 1994.

[22] K. Burke, Some connections between finitely generated functors and pp-types, preprint, University of Manchester, 1997.

[23] K. Burke, A general character theory for modules, Comm. Algebra, 28 (2000), 265-297.

[24] K. Burke, Co-existence of Krull filtrations, J. Pure Appl. Algebra, to appear.

[25] K. Burke, Pure-injective hulls of expanding string modules, University of Manchester, preprint, 1997.

[26] K. Burke and M. Prest, Rings of definable scalars and biendomorphism rings, pp. 188-201 in Model Theory of Groups and Automorphism Groups, London Math. Soc. Lect. Note Ser., Vol. 244, Cambridge University Press, 1997.

[27] K. Burke and M. Prest, The Ziegler and Zariski spectra of some domestic string algebras, Algebras and Representation Theory, to appear.

[28] M. C. R. Butler and C. M. Ringel, Auslander-Reiten sequences with few middle terms and applications to string algebras, Comm. Alg., 15 (1987), 145-179.

[29] C. C. Chang and H. J. Keisler, Model Theory, North-Holland, Amsterdam, 1973 (3rd Ed. 1990).

[30] P. M. Cohn, On the free product of associative rings, Math., Zeitschr. 71 (1959), 380-398.

[31] W. Crawley-Boevey, On tame algebras and bocses, Proc. London Math. Soc., 56 (1988), 451-483. 
[32] W. Crawley-Boevey, Tame algebras and generic modules, Proc. London Math. Soc., 63 (1991), 241-265.

[33] W. Crawley-Boevey, Regular modules for tame hereditary algebras, Proc. London Math. Soc., 62 (1991), 490-508.

[34] W. Crawley-Boevey, Modules of finite length over their endomorphism rings, pp. 127-184 in Representations of Algebras and Related Topics, London Math. Soc. Lecture Note Ser. Vol. 168, Cambridge University Press, 1992.

[35] W. Crawley-Boevey, Locally finitely presented additive categories, Comm. Algebra, 22 (1994), 1641-1674.

[36] W Crawley-Boevey, Infinite-dimensional modules in the representation theory of finite-dimensional algebras, pp. 29-54 in Algebras and Modules I, Canadian Math. Soc. Conf. Proc., Vol. 23, Amer. Math. Soc, 1998.

[37] V. Dlab and C. M. Ringel, Indecomposable representations of graphs and algebras, Mem. Amer. Math. Soc., No. 173, Providence, RI, 1976.

[38] S. Ehrahimi-Atani, On pure-injective modules over pullback rings, Comm. Algebra, to appear.

[39] P. C. Eklof and I. Herzog, Model theory of modules over a serial ring, Ann. Pure Appl. Math., 72 (1995), 145-176.

[40] P. Eklof and G. Sabbagh, Model-completions and modules, Ann. Math. Logic, 2 (1971), 251-295.

[41] A. Facchini and G. Puninksi, $\Sigma$-pure-injective modules over a serial ring, pp. 145-162 in Abelian Groups and Modules, Kluwer, Dordrecht, 1995.

[42] C. Faith, Algebra: Rings, Modules and Categories I, Springer-Verlag, 1973.

[43] E. Fisher, Powers of saturated modules, J. Symbolic Logic, 37 (1972), 777 .

[44] E. Fisher, Abelian Structures, Yale University, 1974/5, partly published as Abelian Structures I, pp. 270-322 in Abelian Group Theory, Lecture Notes in Mathematics, Vol. 616, Springer-Verlag, 1977.

[45] P. Gabriel, Des catégories abéliennes, Bull. Soc. Math. France, 90 (1962), 323-448.

[46] S. Garavaglia, Decomposition of totally transcendental modules, J. Symbolic Logic, 45 (1980), 155-164. 
[47] S. Garavaglia, Dimension and rank in the model theory of modules, preprint, University of Michigan, 1979, revised 1980.

[48] W. Geigle, The Krull-Gabriel dimension of the representation theory of a tame hereditary artin algebra and applications to the structure of exact sequences, Manuscripta Math., 54 (1985), 83-106.

[49] W. Geigle and H. Lenzing, A class of weighted projective lines arising in representation theory of finite-dimensional algebras, pp. 265-297 in Singularities, Representations of Algebras and Vector Bundles, Lecture Notes in Math., Vol. 1273, Springer-Verlag, 1987.

[50] G. Geisler, Zur Modelltheorie von Moduln, Doctoral dissertation, Universität Freiburg, 1994.

[51] Gelfand and Ponomarev, Indecomposable representations of the Lorenz group, Uspehi Mat. Nauk, 23(2) (1968), translated in Russian Math. Surveys, 23(2) (1968), 1-58.

[52] K. R. Goodearl, Von Neumann Regular Rings, Pitman, London, 1979.

[53] K. R. Goodearl, Artinian and noetherian modules over regular rings, Comm. Algebra, 8 (1980), 477-504.

[54] L. Gruson and C. U. Jensen, Modules algébriquement compact et foncteurs $\lim ^{(i)}$, C. R. Acad. Sci. Paris, 276 (1973), 1651-1653.

[55] L. Gruson and C. U. Jensen, Dimensions cohomologiques relièes aux foncteurs $\lim ^{(i)}$, pp. 243-294 in Lecture Notes in Mathematics, Vol. 867, Springer-Verlag, 1981.

[56] R. Hartshorne, Coherent functors, Adv. in Math., 140 (1998), 44-94.

[57] I. Herzog, Modules with few types, J. Algebra, 149 (1992), 358-370.

[58] I. Herzog, Elementary duality of modules, Trans. Amer. Math. Soc., 340 (1993), 37-69.

[59] I. Herzog, The Auslander-Reiten translate, Contemp. Math., 130 (1992), 153-165.

[60] I. Herzog, A test for finite representation type, J. Pure Appl. Algebra, 95 (1994), 151-182.

[61] I. Herzog, Dualizing rings and a characterisation of finite representation type, C. R. Acad. Sci. Paris, 322 (1996), 507-510.

[62] I. Herzog, The Ziegler spectrum of a locally coherent Grothendieck category, Proc. London Math. Soc., 74 (1997), 503-558. 
[63] I. Herzog, The endomorphism ring of a localised coherent functor, J. Algebra, 191 (1997), 416-426.

[64] I. Herzog, Locally simple objects, pp. 341-351 in P. Eklof and R. Göbel (Eds.), Proc. Int. Conf. on Abelian Groups and Modules, Dublin, Birkhauser, 1999.

[65] I. Herzog, The pseudo-finite dimensional representations of $s l(2, k)$, Selecta Mathematica, to appear.

[66] I. Herzog and V. Puninskaya, The model theory of divisible modules over a domain, Fundamentalnaya i Prikladnaya Matematika, 2 (1996), $563-594$.

[67] M. Hochster, Prime ideal structure in commutative rings, Trans. Amer. Math. Soc., 142 (1969), 43-60.

[68] B. Huisgen-Zimmermann, Purity, algebraic compactness, direct sum decompositions and representation type, in [20]

[69] C. U. Jensen and H. Lenzing, Model Theoretic Algebra, Gordon and Breach, 1989.

[70] I. Kaplansky, Infinite Abelian Groups, Univ. of Michigan Press, Ann Arbor, 1954.

[71] Kielpinski, On $\Gamma$-pure injective modules, Bull. Polon. Acad. Sci., 15 (1967), 127-131.

[72] L. Klingler and L. Levy, Wild torsion modules over Weyl algebras and general torsion modules over HNPs, J. Algebra, 172 (1995), 273-300.

[73] H. Krause, The endocategory of a module, pp 419-432 in CMS Conf. Proc. Vol 18, 1996.

[74] H. Krause, The spectrum of a locally coherent category, J. Pure Applied Algebra, 114 (1997), 259-271.

[75] H. Krause, Finitistic dimension and the Ziegler spectrum, Proc. Amer. Math. Soc., 126 (1998), 983-987.

[76] H. Krause, Stable equivalence preserves representation type, Comm. Math. Helv., 72 (1997), 266-284.

[77] H. Krause, The Spectrum of a Module Category, Habilitationsschrift, Universität Bielefeld, 1997, to appear in Mem. Amer. Math. Soc.

[78] H. Krause, Exactly definable categories, J. Algebra, 201 (1998), 456492. 
[79] H. Krause, Generic modules over Artin Algebras, Proc. London Math. Soc., 76 (1998), 276-306.

[80] H. Krause, Functors on locally finitely presented categories, Colloq. Math., 75 (1998), 105-132.

[81] H. Krause, Smashing subcatgories and the telescope conjecture - an algebraic approach, Invent. Math., 139 (2000), 99-133.

[82] H. Krause, Decomposing thick subcategories of the stable module category, Math. Ann. 313 (1999), 95-108.

[83] T. G. Kucera and M. Prest, Imaginary modules, J. Symbolic logic, 57 (1992), 698-723.

[84] L. Levy, Modules over pullbacks and subdirect sums, J. Algebra, 71 (1981), 50-61.

[85] M. Makkai and R. Parè, Accessible Categories: the Foundations of Categorical Model Theory, Comptemp. Math. Vol. 104, Amer. Math. Soc., 1989.

[86] A. Marcja, M. Prest and C. Toffalori, The stability classification for abelian-by-finite groups and modules over a group ring, J. London Math. Soc., 47 (1993), 212-226.

[87] A. Marcja, M. Prest and C. Toffalori, The torsionfree part of the Ziegler spectrum of $R G$ when $R$ is a Dedekind domain and $G$ is a finite group, preprint, 2000.

[88] A. Marcja and C. Toffalori, Decidable representations, J. Pure Applied Algebra, 103 (1995), 189-203.

[89] H. Marubayashi, Modules over bounded Dedekind prime rings II, Osaka J. Math., 9 (1972), 427-445.

[90] E. Matlis, Injective modules over noetherian rings, Pacific J. Math., 8 (1958), 511-528.

[91] B. Mitchell, Rings with several objects, Adv. in Math., 8 (1972), 1-161.

[92] E. Monari-Martinez, On $\Sigma$-pure-injective modules over valuation domains, Arch. Math., 46 (1986), 26-32.

[93] R. S. Pierce, Modules over Commutative Regular Rings, Mem. Amer. Math. Soc., Vol. 70, 1967.

[94] F. Point and M. Prest, Decidability for theories of modules, J. London Math. Soc., 38 (1988), 193-206. 
[95] N. Popescu, Abelian Categories with Applications to Rings and Modules, Academic Press, 1973.

[96] M. Prest, Some model-theoretic aspects of torsion theories, J. Pure Applied Alg., 12 (1978), 295-310.

[97] M. Prest, Some Model-Theoretic Properties of Torsion Theories in Abelian Categories, Doctoral Thesis, University of Leeds, 1979.

[98] M. Prest, Elementary torsion theories and locally finitely presented Abelian categories, J. Pure Applied Algebra, 18 (1980), 205-212.

[99] M. Prest, Tame categories of modules and decidability, University of Liverpool, 1985.

[100] M Prest, Model theory and representation type of algebras, pp.219-260 in Logic Colloquium '86 (Proceedings of the European meeting of the Association for Symbolic Logic, Hull, 1986), North-Holland, Amsterdam, 1988.

[101] M. Prest, Duality and pure-semisimple rings, J. London Math. Soc., 38 (1988), 193-206.

[102] M. Prest, Model Theory and Modules, London Math. Soc. Lecture Notes Ser. Vol. 130, Cambridge University Press, 1988.

[103] M. Prest, Remarks on elementary duality, Ann. Pure Applied Logic, 62 (1993), 183-205.

[104] M. Prest, The (pre-)sheaf of definable scalars, University of Manchester, preprint, 1994.

[105] M. Prest, Tensor product and theories of modules, J. Symbolic Logic, 64 (1999), 617-628.

[106] M. Prest, A note concerning the existence of many indecomposable infinite-dimensional pure-injective modules over some finite-dimensional algebras, preprint, University of Manchester, 1995.

[107] M. Prest, Representation embeddings and the Ziegler spectrum, J. Pure Applied Algebra, 113 (1996), 315-323.

[108] M. Prest, Epimorphisms of rings, interpretations of modules and strictly wild algebras, Comm. Algebra, 24 (1996), 517-531.

[109] M. Prest, Interpreting modules in modules, Ann. Pure Applied Logic, 88 (1997), 193-215. 
[110] M. Prest, Morphisms between finitely presented modules and infinitedimensional representations, pp. 447-455 in Canad. Math. Soc. Conf. Proc. Ser., Vol. 24 (1998).

[111] M. Prest, Ziegler spectra of tame hereditary algebras, J. Algebra, 207 (1998), 146-164.

[112] M. Prest, The Zariski spectrum of the category of finitely presented modules, University of Manchester, preprint, 1998.

[113] M. Prest, The sheaf of locally definable scalars over a ring, pp. 339-351 in Models and Computability, London Math. Soc. Lect. Note Ser., Vol. 259, Cambridge University Press, 1999.

[114] M. Prest, Sheaves of definable scalars over tame hereditary algebras, University of Manchester, preprint, 1998.

[115] M. Prest, Topological and geometric aspects of the Ziegler spectrum, pp. 369-392 in [20].

[116] M. Prest, Model theory for Algebra, this volume.

[117] M. Prest, Ph. Rothmaler and M. Ziegler, Absolutely pure and flat modules and "indiscrete" rings, J. Algebra, 174 (1995), 349-372.

[118] M. Prest, Ph. Rothmaler and M. Ziegler, Extensions of elementary duality, J. Pure Applied Algebra, 93 (1994), 33-56.

[119] M. Prest and V. Puninskaya, Modules with few types over some finitedimensional algebras, J. Symbolic Logic, to appear.

[120] M. Prest and G. Puninski, Some model theory over hereditary noetherian domains, J. Algebra, 211 (1999), 268-297.

[121] M. Prest and G. Puninski, Pure injective hulls of a finite length module over a generalised Weyl algebra, preprint, 2000.

[122] M. Prest and G. Puninski, Pure injective envelopes of finite length modules over a Generalised Weyl Algebra, preprint, University of Manchester, 2000.

[123] V. Puninskaya, Injective minimal modules, Algebra i Logika, 30 (1994), 211-226, transl. Algebra and Logic, 33 (1994), 120-128.

[124] V. Puninskaya, Modules with few types over a hereditary noetherian prime ring, J, Symbolic Logic, to appear.

[125] G. Puninski, Superdecomposable pure-injective modules over commutative valuation rings, Algebra i Logika, 31 (1992), 655-671, transl. Algebra and Logic 31 (1992), 377-386. 
[126] G. Puninski, RD-formulas and W-rings (in Russian), Matematicheskie Zametki, 53 (1993), 95-103.

[127] G. Puninski, On Warfield rings, Algebra i Logika, 33 (1994), 264-285, transl. Algebra and Logic, 33 (1994) 147-159.

[128] G. Puninksi, Indecomposable pure-injective modules over serial rings, Trudy Mosk. mat. ob. 56 (1995), 176-191, transl. Trans. Moscow Math. Soc., 56 (1995), 137-147.

[129] G. Puninski, Pure-injective modules over right noetherian serial rings, Comm. in Algebra, 23 (1995), 1579-1591.

[130] G. Puninski, Cantor-Bendixson rank of the Ziegler spectra over a commutative valuation domain, J. Symbolic Logic, to appear.

[131] G. Puninski, Serial rings, manuscript of book, Moscow State Social University, 1998.

[132] G. Puninski, Finite length and pure-injective modules over a ring of differential operators, J. Algebra, 231 (2000), 546-560.

[133] G. Puninski, Pure-injective and finite length modules over certain rings of differential polynomials, Proc. Moscow-Taiwan Workshop, to appear.

[134] G. Puninski, Pure-injective modules over a commutative valuation domain, preprint, 2000.

[135] G. Puninski, M. Prest and Ph. Rothmaler, Rings described by various purities, Comm. Algebra, 27 (1999), 2127-2162.

[136] G. Puninski and R. Wisbauer, $\Sigma$-injective modules over left duo and left distributive rings, J. Pure Appl. Algebra, 113 (1996), 55-66.

[137] R. Rentschler and P. Gabriel, Sur la dimension des anneaux et ensembles ordonnés, C. R. Acad. Sci. Paris, Ser. A5 (1967), 712-715.

[138] G. Reynders, Ziegler spectra of serial rings with Krull dimension, Comm. Algebra, 27 (1999), 2583-2611.

[139] J. Rickard, Idempotent modules in the stable category, J. London Math. Soc., 56 (1997), 149-170.

[140] C. M. Ringel, Infinite-dimensional representations of finite-dimensional hereditary algebras, Symp. Math. inst. Naz. Alta. Mat., 23 (1979), 321412 .

[141] C. M. Ringel, Tame Algebras and Integral Quadratic Forms, Lecture Notes in Math., Vol. 1099, Springer-Verlag, 1984. 
[142] C. M. Ringel, The indecomposable representations of the dihedral 2groups, Math. Ann., 214 (1975), 19-34.

[143] C. M. Ringel, Some algebraically compact modules I, pp.419-439 in Abelian Groups and Modules, Eds. A. Facchini and C. Menini, Kluwer, 1995.

[144] C. M. Ringel, The Ziegler spectrum of a tame hereditary algebra, Colloq. Math., 76 (1998), 105-115.

[145] C. M. Ringel, Infinite length modules: some examples as introduction, in $[20]$

[146] J.-E. Roos, Locally Noetherian categories and generalised strictly linearly compact rings: Applications, pp. 197-277 in Category Theory, Homology Theory and their Applications, Lecture Notes in Mathematics, Vol. 92, Springer-Verlag, 1969.

[147] Ph. Rothmaler, Some model theory of modules II On stability and categoricity of flat modules, J. Symbolic Logic, 48 (1983), 970-985.

[148] Ph. Rothmaler, Mittag-Leffler Modules, Ann. Pure Applied Logic, 88 (1997), 227-239.

[149] Ph. Rothmaler, Purity in model theory, pp.XX in R. Göbel and XX (Eds.), Advances in Algebra and Model Theory, Gordon and Breach, 1997.

[150] G. Sabbagh, Sur la pureté dans les modules, C. R. Acad. Sci. Paris, 271 (1970), 865-867.

[151] G. Sabbagh, Aspects logiques de la pureté dans les modules, C. R. Acad. Sci. Paris, 271 (1970), 909-912.

[152] G. Sabbagh, Sous-modules purs, existentiellement clos et élémentaires, C. R. Acad. Sci. Paris, 272 (1970), 1289-1292.

[153] G. Sabbagh and P. Eklof, Definability problems for rings and modules, J. Symbolic Logic, 36 (1971), 623-649.

[154] L. Salce, Valuation domains with superdecomposable pure injective modules, pp. 241-245 in Lecture Notes Pure Appl. Math., Vol. 146, Springer-Verlag, 1993.

[155] M. Schmidmeier, Auslander-Reiten-Köcher für artinische Ringe mit Polynomidentität, Diss. Universität Munchen, 1996.

[156] A. Schofield, Representations of Rings over Skew Fields, London Math. Soc. Lecture Notes Ser. Vol. 92, Cambridge University Press, 1985. 
[157] J. Schröer, Hammocks for String Algebras, Habilitationsschrift, Universität Bielefeld, 1998.

[158] J. Schröer, On the Krull-Gabriel dimension of an algebra, Math. Zeit., to appear.

[159] J. Schröer, The Krull-Gabriel dimension of an algebra - open problems, to appear in [20].

[160] S. Shelah, Classification Theory and the Number of Non-Isomorphic Models, North-Holland, 1978.

[161] D. Simson, A class of potential counterexamples to the pure semisimplicity conjecture, pp. 345-373 in Advances in Algebra and Model Theory, Gordon and Breach, 1997.

[162] B. Stenström, Rings of Quotients, Springer-Verlag, 1975.

[163] W. Szmielew, Elementary properties of abelian groups, Fund. Math., 41 (1954), 203-271.

[164] B. R. Tennison, Sheaf Theory, London Math. Soc. Lecture Notes Ser., Vol. 20, Cambridge University Press, 1975.

[165] C. Toffalori, Decidability for modules over a group ring III, Comm. Algebra 24 (1996), 331-344.

[166] C. Toffalori, Wildness implies undecidability for lattices over group rings, J. Symbolic Logic, 62 (1997), 1429-1447.

[167] C. Toffalori, Some decidability questions for lattices over a group ring, preprint, 1997.

[168] C. Toffalori, Decidability for $\mathbb{Z} G$-modules when $G$ is cyclic of prime order, Math. Logic. Quart., 42 (1996), 369-378.

[169] C. Toffalori, Some decidability results for $\mathbb{Z} G$-modules when $G$ is cyclic of squarefree order, Math. Logic. Quart., 42 (1996), 433-445.

[170] J. Trlifaj, Two problems of Ziegler and uniform modules over regular rings, pp. 373-383 in Abelian Groups and Modules, M. Dekker, New York, 1996.

[171] J. Trlifaj, Uniform modules, $\Gamma$-invariants, and Ziegler spectra of regular rings, pp 327-340 in Trends in Mathematics, Birkhäuser, 1999.

[172] L. V. Tyukavkin, Model completeness of certain theories of modules, Algebra i Logika, 21(1) (1982), 73-83, translated in Algebra and Logic, 21(1) (1982), 50-57. 
[173] R. B. Warfield, Purity and algebraic compactness for modules, Pacific J. Math., 28 (1969), 699-710.

[174] R. B. Warfield, Serial rings and finitely presented modules, J. Algebra, 37 (1975), 187-222.

[175] E. Wilkins, An equivalence induced by Ext and Tor applied to the finitistic weak dimension of coherent rings, Glasgow Math. J., 40 (1998), $167-176$.

[176] Wisbauer, Foundations of Module and Ring Theory, Gordon and Breach, Philadelphia, 1991.

[177] D. Zelinsky, Linearly compact rings and modules, Amer. J. Math., 75 (1953), 79-90.

[178] M. Ziegler, Model theory and modules, Ann. Pure Applied Logic, 26 (1984), 149-213.

[179] B. Zimmermann-Huisgen and W. Zimmermann, Algebraically compact rings and modules, Math. Zeit., 161 (1978), 81-93.

[180] B. Zimmermann-Huisgen and W. Zimmermann, On the sparsity of representations of rings of pure global dimension zero, Trans. Amer. Math. Soc., 320 (1990), 695-713.

[181] W. Zimmermann, Rein injektive direkte Summen von moduln, Comm. Algebra, 5 (1977), 1083-1117.

[182] W. Zimmermann, Auslander-Reiten sequences over artinian rings, J. Algebra, 119 (1988), 366-392.

[183] W. Zimmermann, Auslander-Reiten sequences over derivation polynomial rings, J. Pure Appl. Algebra, 74 (1991), 317-332.

[184] W. Zimmermann, Extensions of three classical theorems to modules with maximum condition for finite matrix subgroups, Forum Math., 10 (1998), 377-392.

[185] G. Zwara, Tame algebras and degenerations of modules, to appear in [20]. 